\title{
Comparing nanowire, multijunction, and single junction solar cells in the presence of light trapping
}

\author{
K. R. Catchpole, ${ }^{\text {a) }}$ S. Mokkapati, and F. J. Beck \\ Centre for Sustainable Energy Systems, College of Engineering and Computer Science, \\ Australian National University, ACT 0200, Australia
}

(Received 17 December 2010; accepted 21 March 2011; published online 26 April 2011)

\begin{abstract}
In this paper we quantify the constraints and opportunities for radial junction nanowire solar cells, compared to single junction and multijunction solar cells, when light trapping is included. Both nanowire and multijunction designs are reliant on a very low level of traps in the junction region, and without this, single junction designs are optimal. If low trap density at the junction can be achieved, multijunction cells lead to higher efficiencies than nanowire cells for a given diffusion length, except in the case of submicron diffusion lengths. Thus the radial junction structure is not in itself an advantage in general, though if nanowires allow faster deposition or better light trapping than other structures they could still prove advantageous. (C) 2011 American Institute of Physics. [doi:10.1063/1.3579420]
\end{abstract}

\section{INTRODUCTION}

Nanowire solar cells have recently been the subject of a great deal of investigation. In particular, it has been shown that nanowires can lead to excellent light trapping,,${ }^{1-3}$ high minority carrier diffusion lengths, ${ }^{4}$ and the ability to detach the nanowire structure from the growth substrate. ${ }^{5}$ Kelzenberg et al. have shown for nanowire arrays with a packing fraction around 5\% that close to Lambertian absorption for an equivalent thickness silicon layer can be achieved. ${ }^{1}$ Garnett and Yang have demonstrated path length enhancements of 73 for normal incidence for silicon nanowire arrays on silicon substrates. ${ }^{2}$ Kapadia et al. have performed device modeling showing that efficiencies of $20 \%$ may be possible with $\mathrm{CdS} / \mathrm{CdTe}$ nanowire solar cells, ${ }^{6}$ and promising experimental cell efficiencies have been reported for silicon, ${ }^{7,8}$ III-nitrides, ${ }^{9}$ and for $\mathrm{Si} / \mathrm{GaN}$ heterojunctions. ${ }^{10}$

A related device concept to the nanowire solar cell is the multijunction solar cell. ${ }^{11}$ The multijunction solar cell consists of a number of parallel p-n junctions, with a thickness less than the minority carrier diffusion length of the semiconductor. As with the radial junction nanowire solar cell, this allows efficient carrier collection and hence high short circuit currents for low diffusion lengths. (Note that in the multijunction cells we consider here that all the layers within the device have the same bandgap, unlike in tandem solar cells which are sometimes also referred to as multijunction cells). Both the multijunction and nanowire geometry have higher junction recombination due to the increased junction area, hence a low trap density at the interface is crucial for high efficiencies. ${ }^{12-14}$ Kayes et al. have modeled radial junction nanowire cells compared to single junction cells, neglecting light trapping. ${ }^{14}$ They found a substantial benefit to the nanowire geometry, provided that a low density of traps in the junction region can be achieved. However, Stocks found that, although multijunction solar cells have an advantage

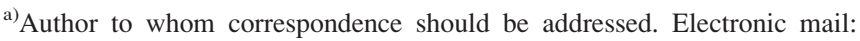
kylie.catchpole@anu.edu.au.
}

over single junction cells when there is no light trapping, when light trapping is included, multijunction cells perform worse than single junction cells. ${ }^{12}$ This comparison was made for the case without a reduced trap density at the interface.

In this paper we compare nanowire, single junction, and multijunction solar cells, taking into account light trapping, for the cases with and without reduced traps at the interface. In doing so, we determine whether there is an intrinsic advantage to the radial nanowire geometry over planar cells.

The model involves the calculation of generation for either a single pass across the device or for an analytical approximation to Lambertian light trapping. This is used with analytical expressions for the collection probabilities to calculate the short-circuit current density. Combined with analytical expressions for the recombination current densities, this allows for the calculation of the J-V curve, and hence the efficiency of each type of device. Figure 1 shows the modeled structures.

In calculating the generation in the device, reflection from the front surface is neglected. For the case without light trapping, it is assume that the light travels a single pass across the device i.e., the absorption $\mathrm{A}$ is given by

$$
A(\lambda)=1-\exp \left(-\alpha f_{\text {area }} W\right)
$$

where $\alpha$ is the absorption coefficient, $W$ is the thickness of the device or the length of the nanowires, and $f_{\text {area }}$ is the area fraction of the nanowires $\left(f_{\text {area }}=1\right.$ for the other types of devices). For nanowires (both the single pass and Lambertian cases), we assume that all incident light is intercepted regardless of the area fraction of the nanowires, and that the absorption depends on the effective thickness $f_{\text {area }} W$. This is consistent with the experimental results of Kelzenberg et al. for light trapping with nanowire arrays. ${ }^{1}$ For the case including light trapping, Lambertian (ergodic) light trapping is assumed i.e., isotropic distribution of the incident light. ${ }^{15}$ This is a standard comparison case for evaluating light 


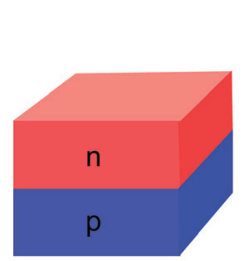

a)

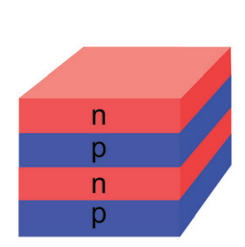

b)

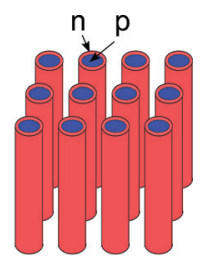

c)
FIG. 1. (Color online) Structures modeled: (a) single junction cell, (b) multijunction cell, and (c) nanowire cell.

trapping schemes, and while it can be exceeded for normal incidence for a number of cases ${ }^{16}$ it has recently been shown that this can only be exceeded for isotropic incidence in very special cases. ${ }^{17}$ We use the analytical expressions for Lambertian light trapping given by Ref. 18

$$
A(\lambda)=\frac{1-R_{b} T^{+} T^{-}}{1-R_{f} T^{+} T^{-}} .
$$

where $R_{b}$ is the reflectance at the rear reflector, $R_{f}$ is the reflectance of the internal upward flux from the front surface, $T^{+}$is the downward flux transmitted to the rear surface, and $T^{-}$is the fraction of light reflected from the rear that is transmitted to the front surface. For Lambertian light trapping we assume zero loss in the rear reflector i.e., $R_{b}=1$, and isotropic distribution of light into the available modes of a bulk device, so that $R_{f}=1-1 / n^{2}$. We also assume randomization of light at both front and rear surfaces, so that $T^{+}=T^{-}=T$. For this case, an effective optical thickness $W_{\text {op }}$ can be defined as $T=\exp \left(-\alpha W_{\text {op }}\right)$ and it can be shown that $W_{\text {op }}$ can be described by a relationship of the form $W_{\text {op }} / W=(2+x) /(1+x)$. The full expressions for $T$ require the evaluation of an infinite series, but a very good approximation can be obtained by using $x=a(\alpha W)^{b}$ with $a=0.935$ and $b=0.67 .{ }^{18}$ The total generation was then calculated by multiplying the wavelength dependent absorption by the photon flux for the AM1.5G spectrum, and integrating over the wavelength range of 300-1300 $\mathrm{nm}$. In order to obtain an analytical expression for the generation for various device thicknesses, the generation for silicon thicknesses of $0.5,1$, $2,5,10,20,50$, and $100 \mu \mathrm{m}$ was fitted to a third-degree polynomial as a function of $\log \left(f_{\text {area }} W\right)$. We also investigate the case of better than Lambertian light trapping, by reducing the light that escapes from the device by setting $R_{f}=1-1 /\left(2 n^{2}\right)$. This has the effect of increasing the path length enhancement in the case of weak absorption by a factor of 2 , from about $4 n^{2} \approx 50$ for a high index material like silicon, to $8 n^{2} \approx 100$.

For nanowires, the collection probabilities were obtained (after some simplification) from Ref. 14

$$
\begin{gathered}
C_{p}=\frac{2 L_{n}^{2}}{L_{p}^{2}} \frac{\beta_{5}}{\beta_{1}^{2}} \frac{I_{1}\left(\beta_{5}\right)}{I_{0}\left(\beta_{5}\right)}, \\
C_{n}=\frac{2 \beta_{2}}{\beta_{1}^{2}}\left[\frac{I_{1}\left(\beta_{1}\right) K_{1}\left(\beta_{2}\right)-K_{1}\left(\beta_{1}\right) I_{1}\left(\beta_{2}\right)}{I_{1}\left(\beta_{1}\right) K_{0}\left(\beta_{2}\right)+K_{1}\left(\beta_{1}\right) I_{0}\left(\beta_{2}\right)}\right],
\end{gathered}
$$

where $I_{n}(x)$ and $K_{n}(x)$ with $n=0$ or 1 are modified Bessel functions of the first or second kind. The other parameters are given by $\beta_{1}=R / L_{p}, \beta_{2}=\left(R-x_{n}\right) / L_{p}$, and $\beta_{5}=x_{p} / L_{n}$. Here, $R$ is the radius of the nanowire, $L_{n}$ and $L_{p}$ are the minority carrier diffusion lengths, and $x_{p}$ and $x_{n}$ are the thicknesses of the p-type and n-type regions, excluding the depletion region. The numbering for $\beta$ has been chosen to be consistent with Ref. 14.

For the single junction, the collection probabilities for the p-type region of each are given by (cf., Ref. 12)

$$
C_{p}=\left(\frac{x_{p}}{W}\right)\left(\frac{L_{n}}{W}\right) \tanh \left(\frac{x_{p}}{L_{n}}\right),
$$

with a corresponding equation for the n-type region. For the multijunction case, with $m$ layers in the device, the collection probability for the p-type layers bounded by two junctions is given by (cf., Ref. 12)

$$
C_{p}=\left[\frac{(m-2) x_{p}}{2 W}\right]\left(\frac{2 L_{n}}{W}\right) \tanh \left(\frac{x_{p}}{2 L_{n}}\right),
$$

again with a corresponding equation for the n-type region. The collection probability for the outermost layers of the multijunction device is given by Eq. (5) and the corresponding n-type equation, except that the thickness for the outermost layers was set to half the thickness of the other layers. It was assumed in each case that all carriers in the depletion region are collected. Note that in all cases, the collection probabilities have been normalized so that if all carriers are collected, the sum of all the collection probabilities across each region would equal 1 .

For the single junction and multijunction cases, the thicknesses of the n-type and p-type regions (neglecting the depletion region) were set so that the collection probability for each region type was approximately equal. For the nanowire case, the radius $R$ was set to equal $L_{n}$, which has previously been found to be close to optimal. ${ }^{14}$

In writing the above equations, we have assumed zero surface recombination. For nanowires, we show later that as long as the surface recombination velocity, $S$, is less than $1000 \mathrm{~cm} / \mathrm{s}$, the effect on efficiency is negligible for minority carrier diffusion lengths of 1 and $10 \mu \mathrm{m}$. This is also the case for single junctions and multijunctions. ${ }^{12}$ It has also been shown that excellent surface passivation $(S=20 \mathrm{~cm} / \mathrm{s})$ can be achieved with nanowires. ${ }^{19}$

The device equations in each case are given by

$$
J=-J_{s c}+f_{\text {area }}\left(\left(J_{B, n}+J_{B, p}\right) \exp \left(\frac{q V}{k T}\right)+J_{\text {dep }}\right)
$$

where $f_{\text {area }}=1$ for the single junction and multijunction cases, and is varied for the nanowire case to reflect the reduced contribution to recombination for reduced area coverage of nanowires. As previously noted, in the case of reduced area fraction the generation for nanowires was calculated for the effective thickness of the nanowires, given by the length of the nanowires multiplied by the area fraction.

The short-circuit current was calculated by multiplying the total generation by the sum of the carrier collection probabilities over each region. That is, it was assumed that generation was distributed uniformly over the device. The 
advantage of the assumption of uniform generation is that it allows an analytical approach to be taken. Non-uniform generation would lead to higher levels of generation and recombination near the front of the device. This is discussed further at the end of this section.

For the single junction and multijunction cases, the equations for the bulk recombination current in the p-type region were ${ }^{12}$

$$
\begin{gathered}
J_{B, p}=\frac{q D_{n} n_{i}^{2}}{L_{n} N_{A}} \tanh \frac{x_{p}}{L_{n}} \text { (single junction), } \\
J_{B, p}=\frac{q D_{n} n_{i}^{2}}{L_{n} N_{A}}\left[\frac{(m-2)}{2} \tanh \frac{x_{p}}{L_{n}}+\tanh \frac{x_{p}}{2 L_{n}}\right] \text { (multi-junction), }
\end{gathered}
$$

with corresponding expressions for the n-type region. For the nanowire case the equations were ${ }^{14}$

$$
\begin{gathered}
J_{B, p}=\frac{2 q D_{n} n_{i}^{2} W}{L_{p}^{2} N_{A}} \frac{\beta_{5}}{\beta_{1}^{2}} \frac{I_{1}\left(\beta_{5}\right)}{I_{0}\left(\beta_{5}\right)}, \\
J_{B, n}=\frac{2 q D_{p} n_{i}^{2} W}{L_{n}^{2} N_{D}} \frac{\beta_{2}}{\beta_{1}^{2}}\left[\frac{I_{1}\left(\beta_{1}\right) K_{1}\left(\beta_{2}\right)-K_{1}\left(\beta_{1}\right) I_{1}\left(\beta_{2}\right)}{I_{1}\left(\beta_{1}\right) K_{0}\left(\beta_{2}\right)+K_{1}\left(\beta_{1}\right) I_{0}\left(\beta_{2}\right)}\right] .
\end{gathered}
$$

For the single junction case, recombination within the depletion region was given by ${ }^{12}$

$$
J_{\mathrm{dep}}=\frac{q n_{i} W}{\sqrt{\tau_{p 0} \tau_{n 0}}} \kappa \sinh (q V / 2 k T),
$$

where $\kappa=(\pi k T) /\left[q\left(V_{\mathrm{bi}}-V\right)\right]$, and where it has been assumed that traps are near midgap. For the multijunction case this was multiplied by the number of junctions. For a normal level of traps within the depletion region, the minority carrier lifetimes within the depletion region were given by $\tau_{n 0}$ $=\tau_{n} /(1+\Gamma)$ and $\tau_{p 0}=\tau_{p} /(1+\Gamma)$, using the trap-assisted tunneling model of Hurkx, ${ }^{20}$ with $\Gamma=2 \sqrt{3}\left(|F| / F_{\Gamma}\right) \exp$ $\left[\left(|F| / F_{\Gamma}\right)^{2}\right], F_{\Gamma}=\left(2 \pi \sqrt{24 m^{*}(k T)^{3}}\right) /(q h), m^{*}=0.25 m_{e}$, and $F=\left(1.5063 \times 10^{-6}\right) q N_{d} / \varepsilon_{n}$. The case of a low level of traps within the depletion region was also modeled, for which it was assumed that $\tau_{n 0}=\tau_{p 0}=1 \mu \mathrm{s}$.

For the nanowires case the corresponding equation is ${ }^{14}$

$$
J_{\text {dep }}=\frac{q n_{i} W}{\sqrt{\tau_{n 0} \tau_{p 0}}} \frac{r_{2}^{2}-r_{1}^{2}}{R^{2}} \sinh \left(\frac{q V}{2 k T}\right)
$$

where $r_{1}=r-\kappa x_{\mathrm{dep}} / 2$ and $r_{2}=r+\kappa x_{\mathrm{dep}} / 2$, with $x_{\mathrm{dep}}$ as the thickness of the depletion region and

$$
r=x_{p}+\frac{\log \left(N_{a} / n_{i p}\right)}{\log \left[\left(N_{a} N_{d}\right) /\left(n_{i p} n_{i n}\right)\right]} x_{\mathrm{dep}} .
$$

Table I lists the parameters and additional equations used in the model. In the model, the minority carrier diffusion length in the p-type region, $L_{n}$, was varied. The minority carrier lifetimes in the n-type and p-type regions were set to be equal i.e., $\tau_{n}=\tau_{p}$, and the minority carrier diffusion length
TABLE I. Additional parameters and equations used in the modeling.

\begin{tabular}{ll}
\hline \hline Parameter/equation & \multicolumn{1}{c}{ Value } \\
\hline$N_{D}, N_{A}$ & $N_{D}=N_{A}=1 \times 10^{18} \mathrm{~cm}^{-3}$ \\
Bandgap narrowing n-type & $\Delta E_{g}=C_{d} \log \left(N / N_{D 0}\right)$ \\
$C_{d}$ & $13.9 \mathrm{meV}$ \\
$N_{D 0}$ & $1.3 \times 10^{17} \mathrm{~cm}^{-3}$ \\
Bandgap narrowing p-type & $\Delta E_{g}=C_{a} \log \left(N / N_{A 0}\right)$ \\
$C_{a}$ & $17.8 \mathrm{meV}^{-3}$ \\
$N_{A 0}$ & $2.3 \times 10^{17} \mathrm{~cm}^{-3}$ \\
Diffusivity & $D_{n}=(k T / q) \mu_{n}, D_{p}=(k T / q) \mu_{p}$ \\
$\mu_{p}$ & $95 \mathrm{~cm}^{2} \mathrm{~V}^{-1} \mathrm{~s}^{-1}$ \\
$\mu_{n}$ & $270 \mathrm{~cm}^{2} \mathrm{~V}^{-1} \mathrm{~s}^{-1}$ \\
\hline \hline
\end{tabular}

in the n-type region, $L_{p}$, was then calculated from $\tau_{p}=L_{p}^{2} / D_{p}$.

For multijunction structures, it was assumed that there are the same number of p-type layers as n-type layers, and the number of layers was then optimized for the Lambertian case with an upper limit of ten layers.

The model was verified by comparing it with the results of Kayes et al. for the single junction and nanowire cases without light trapping, and with Stocks et al. for the cases of single junction and multijunction cells with light trapping. For the comparison with Kayes et al., surface recombination was included to allow a more direct comparison, since analytical expressions that include surface recombination are available for these cases. The efficiencies were compared for a range of diffusion lengths from 0.1 to $10 \mu \mathrm{m}$, along with a normal and low level of traps in the junction, and very good agreement was obtained. The only significant difference was for the efficiencies of very thick single junction cells, where lower efficiencies were calculated compared to the results of Kayes et al. This was due to a reduced collection probability for the uniform generation assumed in this model, compared with generation closer to the junction, as used by Kayes et al. The agreement between the model and the results of Kayes et al. indicates that the assumption of uniform generation does not make a significant difference except in the case of thick single junction cells, which in any case have low collection probabilities for the minority carrier lengths considered here. The comparison with the results of Stocks et al. for single junction and multijunction cells including light trapping also showed excellent agreement.

In the simulations, the efficiency of each type of solar cell was calculated as a function of thickness for a number of different cases: for given minority carrier diffusion lengths, light trapping regimes, area fraction of nanowires, and high or low interface traps.

Figure 2(a) shows efficiency versus thickness for each type of cell when full coverage of nanowires is assumed $\left(f_{\text {area }}=100 \%\right)$ and there is a normal level of traps in the junction region, for $L_{n}=1 \mu \mathrm{m}$. We can see that the maximum efficiency for nanowires is lower than that for multijunction or single junction cells if light-trapping is taken into account. This was also found to be the case for $L_{n}=10 \mu \mathrm{m}$, with a larger relative advantage for single junction and 


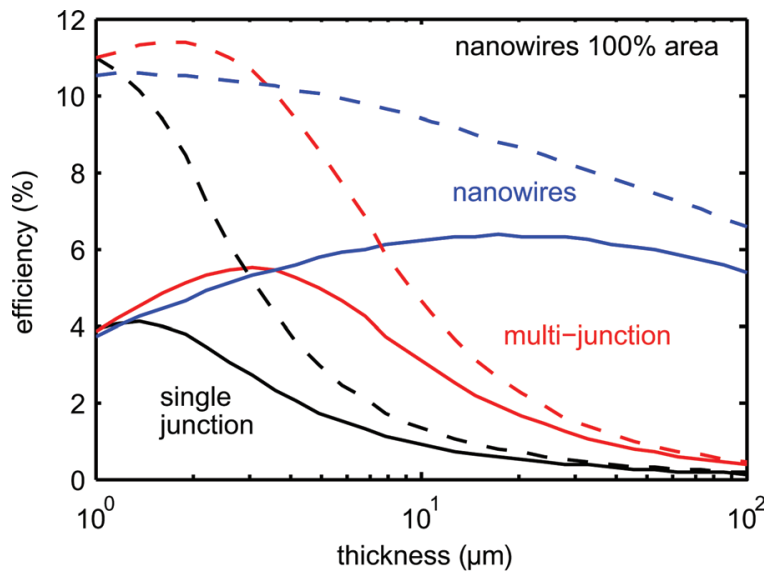

a)

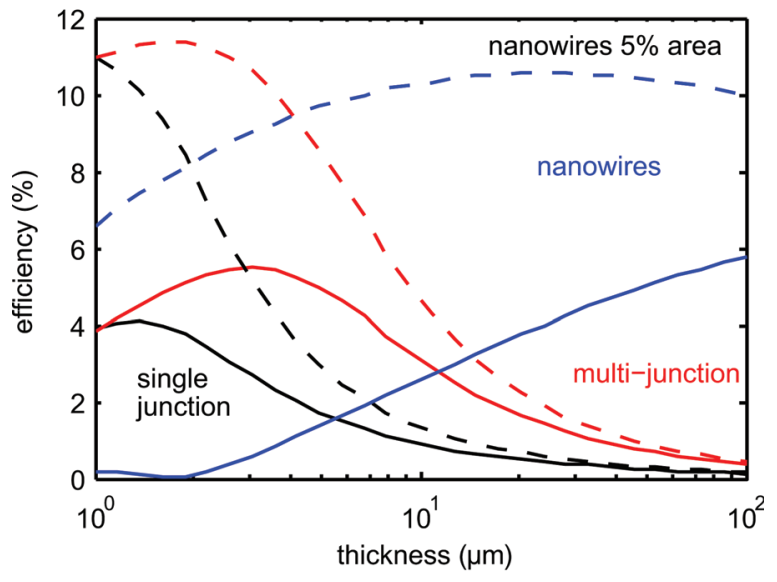

b)

FIG. 2. (Color online) Efficiency vs thickness for the different types of cells for $L_{n}=1 \mu \mathrm{m}$ and a normal level of traps in the junction region. For this case, the optimal value of $m$ for the multijunction cell, including Lambertian light trapping, was 4 . Solid lines are for no light trapping; dashed lines are for Lambertian light trapping. (a) Case of $100 \%$ area fraction of nanowires. (b) Case of $5 \%$ area fraction of nanowires.

multijunction cells in this case. The relatively lower maximum efficiency in Fig. 2(a) is due to increased recombination in the junction region for the nanowires case compared to the other cases.

Figure 2(b) shows the same plots for a 5\% area fraction of nanowires. In this case, the maximum efficiency for the nanowire case has shifted to a higher thickness. Reducing the area fraction decreases the junction recombination at a given thickness, leading to higher efficiencies at greater thicknesses, as compared with Fig. 2(a).

However, the maximum efficiency in Fig. 2(b) of $10.5 \%$ at a nanowire length of $30 \mu \mathrm{m}$ is the same as the maximum efficiency in Fig. 2(a) at a nanowire length of $1.5 \mu \mathrm{m}$. This is because the junction recombination is proportional to $f_{\text {area }}$ and to the length of the nanowire $W$, so the junction recombination for the case of $5 \%$ area fraction and length, $W$, is the same as the junction recombination for the case of $100 \%$ area fraction and length $0.05 \mathrm{~W}$. Since the generation depends also on the effective thickness $=f_{\text {area }} W=0.05 W$ in each case, the efficiency for reduced area fraction of nanowires is shifted with respect to thickness, but does not increase overall. In practice, light trapping may improve for longer

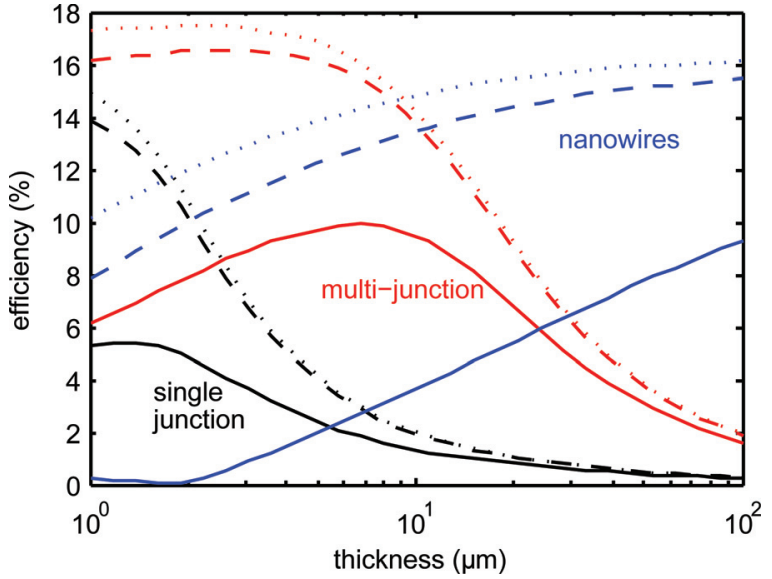

FIG. 3. (Color online) Efficiency vs thickness for the different types of cells for a low level of traps in the junction region. The other parameters were $L_{n}=1 \mu \mathrm{m}, 5 \%$ area fraction, $m=10$. Solid lines are for no light trapping; dashed lines are for Lambertian light trapping; dotted lines are for above Lambertian light trapping.

nanowires, ${ }^{2}$ which would lead to an advantage in reduced area fractions, but the efficiencies would still be lower than for single junction or multijunction cells for a normal level of traps. For $L_{n}=10 \mu \mathrm{m}$ with Lambertian light trapping (not shown), the efficiency of nanowires is still slightly lower $(18.7 \%)$ than the single junction $(20.2 \%)$ or multijunction cases $(20.4 \%$ with $m=4)$ for $5 \%$ area fraction and a normal level of traps.

Figure 3 shows efficiency versus thickness for the three cell types for 5\% area coverage when there is a low level of traps in the junction region. In this case, the nanowire and multijunction structures have a significant efficiency advantage over the single junction structure. However, the multijunction has the highest overall efficiency. In Fig. 3 the multijunction case uses ten layers, but the maximum efficiency of the multijunction case is as high as or higher than the nanowire case for four or more layers. From Fig. 3, it can also be seen that the maximum efficiency for the nanowire structure occurs at a height of over $30 \mu \mathrm{m}$, whereas the maximum efficiency for the multijunction structure occurs for a thickness of 2-3 $\mu \mathrm{m}$. This would suggest that deposition times would be lower for multijunction structures than for nanowire structures for a given deposition rate. (Since most nanowire structures are grown by a catalytic growth under a metal nanoparticle, growth time is proportional to height rather than volume). As shown in Fig. 2, it would be possible to reduce the nanowire height required by increasing the nanowire density. However, the overall efficiency advantage of about $0.8 \%$ for the multijunction structure from Fig. 3 would be maintained. The increased efficiency of the multijunction structure compared to the nanowire structure shows that a moderate rather than a large increase in junction area is desirable for improving the overall device efficiency for diffusion lengths around $1 \mu \mathrm{m}$ (again assuming that a low trap density at the junction is achieved).

Figure 3 also includes the cases of above Lambertian light trapping (dotted lines). This shows that the efficiency advantage to be gained from above Lambertian light trapping is relatively small. In practice, above Lambertian light 


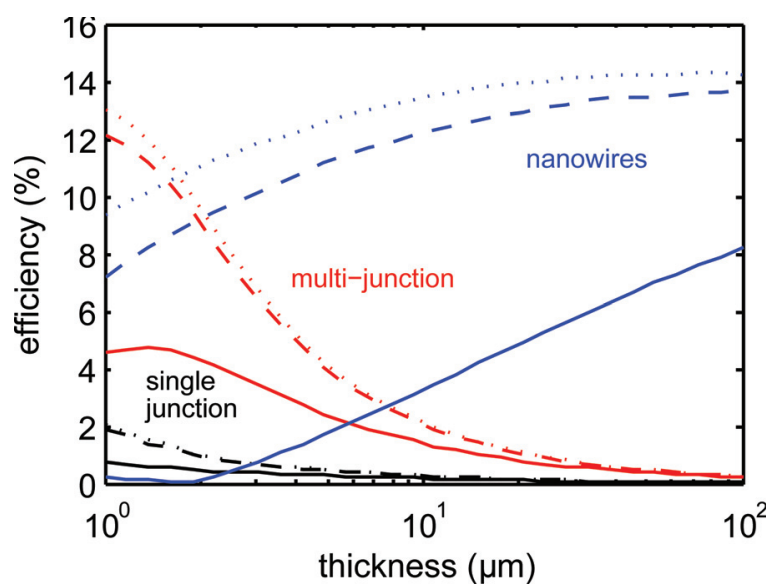

FIG. 4. (Color online) Efficiency vs thickness for the different types of cells for $L_{n}=0.1 \mu \mathrm{m}$, low traps in the junction, $5 \%$ area fraction of nanowires, and $m=10$ for the multijunction. Solid lines are for no light trapping; dashed lines are for Lambertian light trapping; dotted lines are for above Lambertian light trapping.

trapping in a device is likely to be difficult to achieve because along with excellent light confinement it also requires extremely low losses due to, for example, the contacts and free carrier absorption in the semiconductor layers. ${ }^{21}$

For $L_{n}=10 \mu \mathrm{m}$ with Lambertian light trapping and a low level of traps (not shown), the maximum efficiency of nanowires is lower $(19.3 \%)$ than the single junction $(20.6 \%)$ or multijunction cases $(21.3 \%$ with $m=4)$.

Figure 4 shows the efficiency versus thickness for the case of $L_{n}=0.1 \mu \mathrm{m}$, with a $5 \%$ area fraction of nanowires. In this case nanowires have an advantage. For a low level of traps and $L_{n}=0.1 \mu \mathrm{m}$, nanowires have substantially higher efficiency than multijunction cells for $m=10$. The efficiencies are similar for $m=16$ (not shown), but such a large number of layers may be impractical. Silicon nanowires have already demonstrated diffusion lengths of 2-10 $\mu \mathrm{m}$, so this case is of less interest for silicon. However, it may be an interesting structure for novel materials such as the earth abundant semiconductors, e.g., $\mathrm{CuO}$, which have very low diffusion lengths. This would require low levels of traps at the junction to be achieved for these materials. Nanowires are also more efficient than the single junction or multijunction structures for $L_{n}=0.1 \mu \mathrm{m}$ and a normal level of traps (not shown), but in this case the maximum efficiency is very low (3\% with light trapping). For light trapping the photonic properties of the nanowires would need to be maintained i.e., it would be necessary that the nanowires can support confined optical modes at the wavelengths required. This would probably require nanowire diameters of at least half the wavelength of light within the nanowire, i.e., at least $\lambda /(2 n)$ $\approx 150 \mathrm{~nm}$.

Surface recombination has not been included in this analysis in order to retain a simple analytical approach. However, it has been shown that, provided a reasonable level of surface passivation is achieved $(S<1000 \mathrm{~cm} / \mathrm{s}$ ), surface passivation has a negligible effect on the efficiency for the planar and multijunction cases. ${ }^{12} \mathrm{We}$ have calculated the effect of surface recombination for the nanowires case without light trapping, and the same conclusion holds with a maximum efficiency loss of $0.02 \%$ when $S=1000 \mathrm{~cm} / \mathrm{s}$ for $L_{n}=1 \mu \mathrm{m}$ and $0.2 \%$ when $L_{n}=10 \mu \mathrm{m}$. Therefore neglecting surface recombination is a valid assumption for the structures considered here.

The main advantages of the nanowire structure would be high optical absorption and possibly fast deposition. The radial junction structure is not in itself an advantage, except in the case of very low diffusion lengths and low traps at the junction. Therefore, for relatively large diffusion lengths, nonradial structures should also be considered, although in this case surface recombination will be a critical consideration.

For a normal level of traps, there is no efficiency advantage for a nanowire cell over a single junction cell, for minority carrier diffusion lengths of $1-10 \mu \mathrm{m}$, assuming Lambertian light trapping, regardless of the packing fraction. For a low level of traps, nanowires have an advantage over single junction cells for $L_{n}=1 \mu \mathrm{m}$, but multijunction cells are better, assuming Lambertian light-trapping. For $L_{n}=10$ $\mu \mathrm{m}$ and low traps, single junctions and multijunctions are better than nanowires. Thus the radial junction structure is not in itself an advantage except in the case of very low diffusion length and low traps at the junction.

\section{ACKNOWLEDGMENTS}

This work was supported by the Australian Research Council.

${ }^{1}$ M. D. Kelzenberg, S. W. Boettcher, J. A. Petykiewicz, D. B. TurnerEvans, M. C. Putnam, E. L. Warren, J. M. Spurgeon, R. M. Briggs, N. S. Lewis, and H. A. Atwater, Nature Mater. 9, 239 (2010).

${ }^{2}$ E. Garnett, and P. Yang, P. Nano Lett. 10, 1082 (2010).

${ }^{3}$ O. L. Muskens, J. G. Rivas, R. E. Algra, E. P. A. M. Bakkers, and A. Lagendijk, Nano Lett. 8(9), 2638(2008).

${ }^{4}$ M. C. Putnam, D. B. Turner-Evans, M. D. Kelzenberg, S. W. Boettcher, N. S. Lewis, and H. A. Atwater, Appl. Phys. Lett. 95, 163116 (2009).

${ }^{5}$ K. E. Plass, M. A. Filler, J. M. Spurgeon, B. M. Kayes, S. Maldonado, B. S. Brunschwig, H. A. Atwater, and N. S. Lewis, Adv. Mater. 21, 325 (2009).

${ }^{6}$ R. Kapadia, Z. Fan, and A. Javey, Appl. Phys. Lett. 96, 103116 (2010).

${ }^{7}$ B. Tian, X. Zheng, T. J. Kempa, Y. Fang, N. Yu, G. Yu, J. Huang, and C. M. Lieber, Nature (London) 449(7164), 885(2007).

${ }^{8}$ V. Sivakov, G. Andrä, A. Gawlik, A. Berger, J. Plentz, F. Falk, and S. H. Christiansen, Nano Letters 9(4), 1549(2009).

${ }^{9}$ Y. Dong, B. Tian, T. J. Kempa, and C. M. Lieber, Nano Lett. 9(5), 2183 (2009).

${ }^{10}$ Y. B. Tang, Z. H. Chen, H. S. Song, C. S. Lee, H. T. Cong, H. M. Cheng, W. J. Zhang, I. Bello, and S. T. Lee, Nano Lett. 8(12), 4191 (2008).

${ }^{11}$ M. A. Green, and S. R. Wenham, Appl. Phys. Lett. 65, 2907 (1994).

${ }^{12}$ M. J. Stocks, A. Cuevas, and A. W. Blakers, Progr. Photovoltaics 4, 35 (1996).

${ }^{13}$ M. A. Green, Prog. Photovoltaics 4, 375 (1998).

${ }^{14}$ B. M. Kayes, H. A. Atwater, Hand N. S. Lewis, J. Appl. Phys. 97, 114302 (2005).

${ }^{15}$ E. Yablonovitch, J. Opt. Soc. Am. 72(7), 899 (1982).

${ }^{16}$ P. Campbell, and M. A. Green, J. Appl. Phys. 62(1), 243 (1987).

${ }^{17}$ Z. Yu, A. Raman, and S. Fan, Proc. Natl. Acad. Sci. U.S.A. 107, 17491 (2010).

${ }^{18}$ M. A. Green, Prog. Photovoltaics 10, 235 (2002).

${ }^{19}$ O. Demichel, V. Calvo, A. Besson, P. Noe, B. Salem, N. Pauc, F. Oehler, P. Gentile, and N. Magnea, Nano Lett. 10, 2323 (2010).

${ }^{20}$ G. A. M. Hurkx, D. B. M. Klaassen, and M. P. G. Knuvers, IEEE Trans. Electron. Dev. 39, 331 (1992).

${ }^{21}$ J. Gee, "The effect of parasitic absorption on light trapping in thin silicon solar cells," in Twentieth IEEE Photovoltaic Specialists Conference (1988), p. 549. 